\title{
SELF-ASSEMBLY OF TRIBLOCK COPOLYMERS IN AQUEOUS SOLUTION
}

\author{
BRUNO URBANO ${ }^{1}$, PATRICIO SILVA ${ }^{1}$, ANDRÉS F. OLEA ${ }^{1 *}$, IRMA FUENTES ${ }^{2}$, FRANCISCO MARTINEZ ${ }^{2}$
}

\begin{abstract}
1- Departamento de Ciencias Químicas, Facultad de Ecología y Recursos Naturales, Universidad Andrés Bello, República 275, Santiago de Chile, Chile
2- Departamento de Ciencias de los Materiales, Facultad de Ciencias Físicas y Matemáticas, Universidad de Chile, Av.Tupper 206, Santiago, Chile.
\end{abstract} (Received: 3 April 2008 - Accepted: 7 May 2008)

\begin{abstract}
The aggregation of PE4VP-b-PS-b-PE4VP block copolymers was studied in aqueous solution. Triblock copolymers P4VP-b-PS-b-P4VP were synthesized by sequential anionic polymerization of poly(styrene) and poly(4-vinylpirydine) using sodium naphthalene as a bifunctional initiator. Subsequently, the 4vinylpyridine units were quaternized with ethyl bromide to obtain cationic PE4VP-b-PS-b-PE4VP block copolymers. Both star and crew-cut micelles were formed. The concentrations at which micelles are formed cme were determined, by steady-state and time-resolved fluorescence probing methods, as a function of quaternization degree. The results indicate that cmc of crew-cut micelles increases with increasing charge density of the PE4VP blocks. For star micelles there is not a clear dependency of $\mathrm{cmc}$ with the percentage of quaternization. The lifetime of pyrene fluorescence and the ratio $\mathrm{I}_{1} / \mathrm{I}_{3}$ were determined at concentrations of copolymers well above the $\mathrm{cmc}$, and the results show that the location of pyrene into the micelle changes with the charge density of the micelle corona. The micropolarity sensed by pyrene decreases with increasing quaternization degree. The presence of aggregates was confirmed by transmission electronic microscopy.
\end{abstract}

Keywords: Triblock copolymers, critical micelle concentration, micelles, fluorescence probing methods, pyrene.

\section{INTRODUCTION}

The self-assembling phenomena have been an important area of research in colloid science for many years ${ }^{1-4}$. Initially, low molecular weight amphiphiles were studied extensively, but in the last decades considerable attention has been devoted to amphiphilic block copolymers ${ }^{5}$. The self-assembly of amphiphilic block copolymers in selective solvents can result in the formation of a wide variety of aggregates, such as spheres, rods, and bilayers ${ }^{6}$. The specific morphology of the aggregate can be attributed to a balance of three contributions to the free energy of the system, namely, chain stretching in the core, the interfacial energy, and repulsion between corona chains ${ }^{7}$. There are many factors that affect the above three terms. For example, the block length of the copolymer ${ }^{8}$, the concentration of the precipitant, the initial concentration of polymer $^{9}$, the presence of additives ${ }^{10}$, the common solvent used ${ }^{11}$.

The type of micelle, formed in aqueous solution, depends on the relative proportion of the soluble and insoluble block in the polymer. In the case that the hydrophilic block is larger than the hydrophobic block, i.e. the core is short compared with the diameter of the corona; a "star" micelle is formed. On the other hand, if the hydrophobic block length is larger than the length of the hydrophilic block, a "crew cut" micelle is formed.

Aggregates of block copolymers in solution have potential industrial applications in fields, such as drug delivery ${ }^{12,13}$, cosmetic industry, catalysis, separations, and others ${ }^{14}$.

The aim of the present work is to study the self-assembly of cationic block copolymers in aqueous solution, as a function of block size and charge density on the hydrophilic block. For this purpose a variety of triblock copolymers, based on 4-vinylpyridine and styrene has been synthesized, with different lengths of P4VP and PS blocks. A subsequent reaction of the pyridine group with ethyl bromide has been used to generate a positively charged polymer block (PE4VP). The charge density on the PE4VP block has been tailored by allowing this reaction to proceed to different percentages of quaternization. The $\mathrm{cmc}$ and hydrophobicity of polymer micelles formed in aqueous solution were determined by fluorescence methods using pyrene as a probe. The morphology of the aggregates was determined by transmission electronic microscopy (TEM).

\section{EXPERIMENTAL}

Materials. Styrene (Hoechst) and 4-vinylpyridine (Fluka) were dried by stirring over $\mathrm{CaH}_{2}$ overnight, and distillated under reduced pressure. Naphthalene (Aldrich) was sublimed twice before preparing the initiator. THF was refluxed in presence of metallic sodium and benzophenone, until a blue coloration was obtained. Pyrene (Aldrich) was used as received.

Block copolymers of styrene and 4-vinylpyridine were synthesized by sequential anionic polymerization using sodium naphthalene as a bifunctional initiator ${ }^{14}$. The polymerization was carried out under inert atmosphere in a previous flamed glass reactor. A typical reaction is described for the synthesis of P4VP-b-PS-b-P4VP. The initiator, dissolved in $30 \mathrm{~mL}$ of dry THF, is introduced into the reactor by using a syringe. After the solution has been cooled to $-78^{\circ} \mathrm{C}$, freshly distilled styrene was added, and the solution became yellow, which is the typical color of living polystyryl anions. The polymerization was allowed to proceed for $10 \mathrm{~min}$. After that, an aliquot of the reaction medium was withdrawn for analysis by size exclusion chromatography (SEC), in order to determine the degree of polymerization of the PS block. Subsequently, 4vinylpyridine is added and, immediately the solution color turns from yellow to orange. The polymerization is allowed to proceed for another 15 minutes at $-78^{\circ} \mathrm{C}$, and then stopped by adding methanol to quench the reaction and to precipitate the block copolymer. The 4-vinylpyridine units were quaternized with ethyl bromide to obtain cationic PE4VP-b-PS-b-PE4VP block copolymers. The reaction was carried out in chloroform at $70^{\circ} \mathrm{C}$ for up to 5 days, to obtain PE4VP-b-PS-b-PE4VP with different percentages of quaternization of the P4VP block.

Characterization. Size exclusion chromatography (SEC) was carried out with a Shimadzu LC-20AD apparatus equipped with a MZ-Gel SDplus column. Standard polystyrenes were used for calibration; the number-and weight-average molecular weights and the polydispersity index of the polystyrene block were calculated using GPC Class VIP software. The composition was determined by FTIR and ${ }^{1} \mathrm{H}-\mathrm{NMR}$ measurements, using a Shimadzu FTIR spectrophotometer, and a Bruker Avance 400 spectrometer. The degree of quaternization of P4VP blocks was determined by IR spectroscopy using characteristic bands of absorption of quaternized $\left(1640 \mathrm{~cm}^{-1}\right)$ and nonquaternized $\left(1600 \mathrm{~cm}^{-1}\right)$ repeating units.

Preparation of aqueous dispersion of block copolymers ${ }^{15}$. Aqueous dispersion of triblock copolymers, PE4VP-b-PS-b-PE4VP, were prepared using dialysis method (TB01 and TB02) and direct dissolution (TB03). In the dialysis method samples of polymer were initially dissolved in dimethylformamide. After that, water is added slowly at controlled rate until the solution becomes turbid. At this concentration of water, named cac, formation of polymer aggregates has started. In order to fix the aggregate morphology an excess of water is added after a time called template time. Finally the mixture is dialyzed against distilled water using membrane tubing of 12000 dalton molecular weight cut off. The concentration of PE4VP-b-PS-b-PE4VP after dialysis was determined by liophilization.

Measurement of cmc. The solutions for fluorescence probing studies were prepared by adding $3 \mu \mathrm{L}$ of a pyrene stock solution in THF to the aqueous copolymer solution. The injected volume is less than $0.1 \%$ of the volume solution, and this small amount of THF has no effect on the measured properties.

The critical micelle concentration of copolymers was determined by photophysical methods, and using pyrene as fluorescence probe ${ }^{16}$. The fluorescence and excitation spectra were recorded by using a SLM Aminco SPF500C spectrofluorometer. For the emission, pyrene was excited at $337 \mathrm{~nm}$ and the spectrum was registered between 350 and $500 \mathrm{~nm}$. In the excitation mode, pyrene emission was fixed at $390 \mathrm{~nm}$ and the excitation wavelength was scanned between 300 and $350 \mathrm{~nm}$. All steady-state fluorescence measurements 
were carried out at $25^{\circ} \mathrm{C}$ on air-saturated systems.

The lifetimes of pyrene were determined from the fluorescence decay of the singlet excited pyrene, using a laser system that has been described in detail elsewhere ${ }^{17,18}$.

Micropolarity of copolymer micelles. The ratio $I_{1} / I_{3}$ is obtained from the pyrene fluorescence spectrum, where $I_{1}$ and $I_{3}$ are the intensities of the vibronic bands appearing at 378 and $390 \mathrm{~nm}$ has been used as a measure of the micropolarity. In a similar way, the ratio $\mathrm{I}_{333} / \mathrm{I}_{338}$ was obtained from the excitation spectrum of pyrene. Transmission electronic microscopy was performance on a Joel microscope.

\section{RESULTS AND DISCUSSION}

The formation of a triblock copolymer structure was confirmed by FTIR, ${ }^{1} \mathrm{H}-\mathrm{NMR}$, and GPC. The comparison of IR spectra of PS homopolymer and P4VP-b-PS-b-P4VP shows in the latter the characteristic absorption bands of pyridine. In addition, the chromatogram of the copolymer shows just one peak, at an elution time lower than that observed for PS. The molecular weight and polydispersity index, PI, of the block copolymers obtained by GPC are given in table 1 .

Table 1. Molecular weight and polydispersity index of triblock copolymers, obtained by GPC.

\begin{tabular}{|c|c|c|c|c|c|c|}
\hline \multicolumn{2}{|c|}{ Sample } & \multicolumn{2}{c|}{ Homopolymer } & \multicolumn{2}{c|}{ Copolymer } & \\
\hline$N^{\circ}$ & Composition & PMn & PMw & PMn & PMw & PI \\
\hline TB01 & $\begin{array}{c}\text { P4VP-PS-P4VP } \\
67 / 106 / 67\end{array}$ & 11000 & 15000 & 25000 & 27000 & 1,011 \\
\hline TB02 & $\begin{array}{c}\text { P4VP-PS-P4VP } \\
100 / 221 / 100\end{array}$ & 23000 & 26000 & 44000 & 45000 & 1,009 \\
\hline TB03 & $\begin{array}{c}\text { P4VP-PS-P4VP } \\
104 / 30 / 104\end{array}$ & 3100 & 4000 & 25000 & 30200 & 1,23 \\
\hline
\end{tabular}

The PI values range from 1.001 to 1.230 , indicating that the distribution of molecular weights is quite narrow. This result is typical for anionic living polymerization. The composition of triblock copolymers is denoted as $\mathrm{P}_{\mathrm{V}} \mathrm{VP}_{x}-$ b-PS -P4VP, where $x$ and $y$ represent the number of repetitive units forming the 4-vinylpyridine and styrene blocks, respectively. Both $x$ and $y$ are obtained from the average number molecular weight of the respective block. The data from table 1 shows that the method of synthesis allows tailoring of triblock copolymer structure. For example, copolymers TB01 and TB02 were designed with a large PS block compared to the P4VP blocks, while in TB03 the P4VP blocks are larger than the PS block.

To increase the amphiphilic character of P4VP-b-PS-b-P4VP copolymers, a formal charge has been introduced into the P4VP block by reaction of pyridine ring with ethyl bromide. This reaction was carried out in a way to get different degrees of substituted pyridine. The fraction of pyridine ring converted to ethylpyridinium was determined by FTIR, and ranges from $30 \%$ to $98 \%$ (see table 2). Therefore, the resulting polymers, denoted as PE4VP-b-PS-b-PE4VP, are ionomers carrying different number of charges on the PE4VP blocks.

Table 2. Degree of quaternization of P4VP blocks in P4VP-b-PS-bP4VP.

\begin{tabular}{|c|c|c|}
\hline Sample & Composition & Degree of Quaternization (\%) \\
\hline TB01 & $\begin{array}{c}\text { PE4VP-PS-PE4VP } \\
67 / 106 / 67\end{array}$ & $30-55-65-98$ \\
\hline TB02 & $\begin{array}{c}\text { PE4VP-PS-PE4VP } \\
100 / 221 / 100\end{array}$ & $30-50-75$ \\
\hline TB03 & $\begin{array}{c}\text { PE4VP-PS-PE4VP } \\
104 / 30 / 104\end{array}$ & $30-45-70-80$ \\
\hline
\end{tabular}

Self-assembly of triblock copolymers in aqueous solution. The selfassembling of the aqueous dispersion of amphiphilic block copolymers has been studied by fluorescence spectroscopy using pyrene as a probe. Pyrene has been widely used in the study of microheterogeneous systems because of its photophysical behavior is highly dependent on the environment where it is located ${ }^{19}$. In aqueous solutions of micelles or other similar microaggregates pyrene has been used to report on the micropolarity of the aggregates ${ }^{20}$, and to determine the concentration at which the aggregates are formed ${ }^{21}$. For this, the changes of the fluorescence and excitation spectra, and the lifetime of fluorescence can be used. The emission and excitation spectra of pyrene, in presence of different concentrations of PE4VP ${ }_{104}-\mathrm{PS}_{30}-\mathrm{PE} 4 \mathrm{VP}{ }_{104}$, are shown in Figures 1 and 2, respectively.

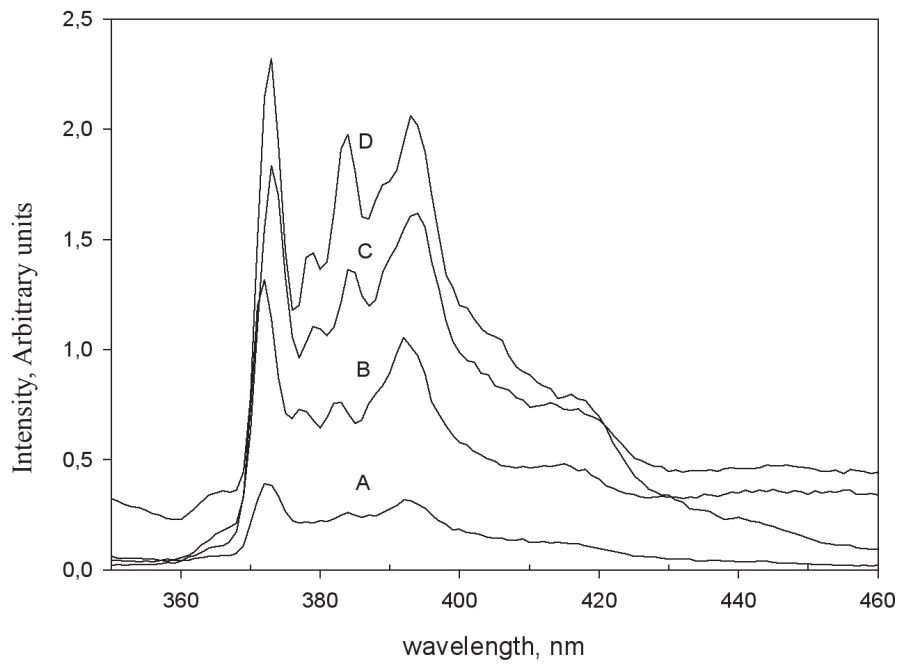

Figure 1. Fluorescence spectra of pyrene in aqueous solution of PE4VP ${ }^{-}$ $\mathrm{PS}_{30}-\mathrm{PE} 4 \mathrm{VP}_{104}$ at different concentration: $\boldsymbol{A} 0 \mathrm{~g} / \mathrm{L} ; \boldsymbol{B} 0.021 \mathrm{~g} / \mathrm{L} ; \boldsymbol{C} 0.043 \mathrm{~g} / \mathrm{L}$; D $0.081 \mathrm{~g} / \mathrm{L} . \lambda_{\mathrm{ex}}=337 \mathrm{~nm}$.

In Figure 1 it can be seen that increase of polymer concentration brings about changes both in the intensity and vibrational structure of the fluorescence spectra of pyrene. The ratio $\mathrm{I}_{1} / \mathrm{I}_{3}$, that corresponds to the ratio of the intensities of the bands observed at $372 \mathrm{~nm}$ and $384 \mathrm{~nm}$, decrease with increasing polymer concentration, whereas the opposite effect is observed for the intensity. On the other hand, the excitation spectra showed in figure 2, exhibit a red shift of the band $(0,0)$ from 333 to $338 \mathrm{~nm}$ upon the increment of block copolymer concentration.

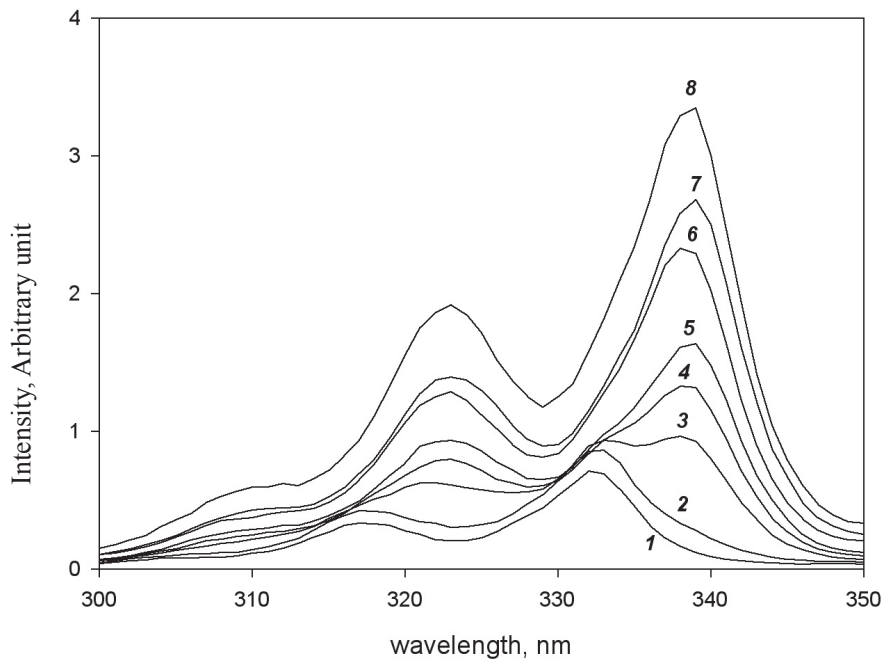

Figure 2. Excitation spectra of pyrene in presence of different concentrations of PE4VP $-\mathrm{PS}_{30}-\mathrm{PE} 4 \mathrm{VP}{ }_{104}: 10 \mathrm{~g} / \mathrm{L} ; 20.021 \mathrm{~g} / \mathrm{L} ; 30.087 \mathrm{~g} / \mathrm{L}$; $40.175 \mathrm{~g} / \mathrm{L} ; 50.350 \mathrm{~g} / \mathrm{L} ; \boldsymbol{6} 0.700 \mathrm{~g} / \mathrm{L} ; 70.100 \mathrm{~g} / \mathrm{L} ; \boldsymbol{8} 1.40 \mathrm{~g} / \mathrm{L} . \lambda_{\mathrm{em}}=390 \mathrm{~nm}$.

All these changes can be used to examine the transfer of pyrene from the aqueous phase to the hydrophobic environment provided by the polymer aggregates. To evaluate the critical micelle concentration $\mathrm{cmc}$ for block copolymers plots of intensity, ratio $\mathrm{I}_{1} / \mathrm{I}_{3}$, lifetime, and ratio $\mathrm{I}_{338} / \mathrm{I}_{333}$ 
against polymer concentration have been used. However, the most accepted photophysical method is that proposed by Wilhelm et al., which gives the "true" $\mathrm{cmc}^{22}$. Basically, this method consists in determining the distribution of pyrene between the micellar phase and the aqueous phase by measuring the extreme values of the ratio $\mathrm{I}_{338} / \mathrm{I}_{333}$ in presence of copolymer. According to this method the concentration of pyrene solubilized into the micelle over the concentration of pyrene in the aqueous phase is given by

$$
\frac{[P y]_{n}}{[P y]_{v}}=\frac{F-F_{\min }}{F_{\max }-F}
$$

where $\mathrm{F}=\mathrm{I}_{338} / \mathrm{I}_{333}$ and $\mathrm{F}_{\text {min }}$ and $\mathrm{F}_{\text {max }}$ are the values of this ratio at low and high concentrations of copolymer. In figure 3 are shown the results obtained by plotting Wilhelm's equation and fluorescence lifetime as a function of

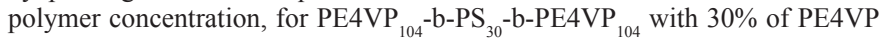
quaternized.

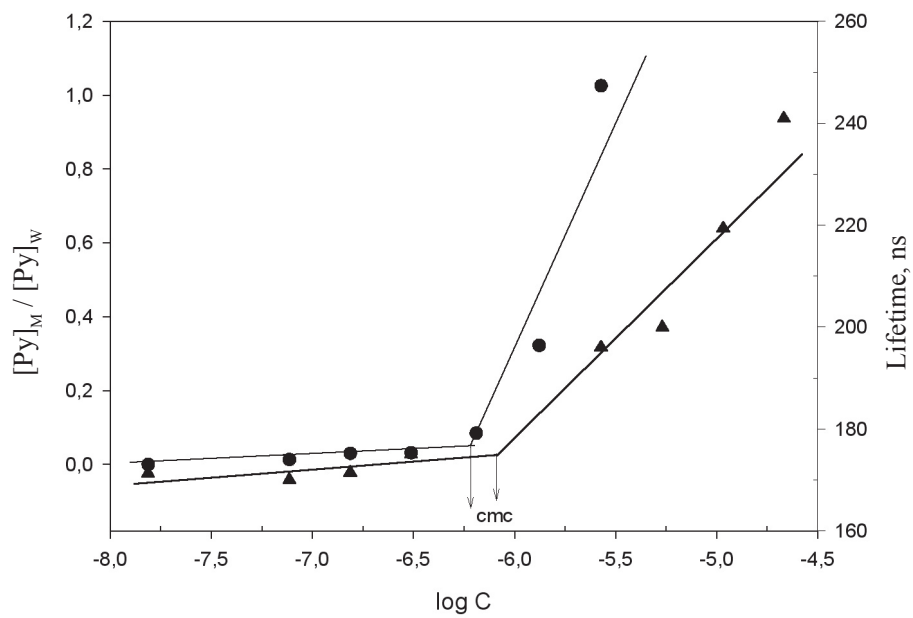

Figure 3. Plots of Wilhelm's equation ( $\left.[\mathrm{Py}]_{\mathrm{W}} /[\mathrm{Py}]_{\mathrm{W}}\right) \mathbf{\Delta}$, and fluorescence lifetime, as a function of concentration of PE4VP ${ }_{104}-\mathrm{b}-\mathrm{PS}_{30}-\mathrm{b}-\mathrm{PE} 4 \mathrm{VP}_{104}$ with $30 \%$ of quaternization.

The cmc values obtained with both methods for this block copolymer with different degrees of quaternization are given in table 3 .

Table 3. Values of cmc of PE4VP ${ }_{104}-\mathrm{b}-\mathrm{PS}_{30}-\mathrm{b}-\mathrm{PE} 4 \mathrm{VP}_{104}$ obtained with Wilhelm's method, and by fluorescence lifetime.

\begin{tabular}{|c|c|c|c|}
\hline Copolymer & $\begin{array}{c}\text { \% of } \\
\text { quaternization }\end{array}$ & $\begin{array}{c}\text { cmc, } \mu \mathrm{M} \\
\text { (Wilhem) }\end{array}$ & $\begin{array}{c}\text { cmc, } \mu \mathrm{M} \\
\text { (lifetime) }\end{array}$ \\
\hline \multirow{3}{*}{$\begin{array}{c}\text { PE4VP-PS-PE4VP } \\
\text { 104/30/104 }\end{array}$} & 30 & 0.63 & 0.89 \\
\cline { 2 - 4 } & 45 & 0.20 & 0.40 \\
\cline { 2 - 4 } & 70 & 0.40 & 0.56 \\
\cline { 2 - 4 } & 80 & 0.58 & 1.12 \\
\hline
\end{tabular}

The data shows that the cmc values are in the order of $10^{-7} \mathrm{M}$, but there is not a clear relationship between the charge density on the polymer chain and the $\mathrm{cmc}$. The $\mathrm{cmc}$ obtained from the fluorescence lifetime are higher than those obtained by Wilhelm's method, but follow the same tendency. For diblock copolymers PS ${ }_{100}$-b-PE4VP 100 and $\mathrm{PS}_{100}$-b-PE4VP ${ }_{490}$, cmc values of $4.6 \times 10^{-7}$ to $2.2 \times 10^{-6} \mathrm{M}$ have been reported, respectively. ${ }^{23}$

Aqueous solutions of copolymers that form crew-cut micelles are turbid, and therefore Wilhelm's method cannot be used. For these systems we have used the fluorescence lifetime of pyrene to determine the $\mathrm{cmc}$. A typical plot is shown in figure 4 and the cmc values are listed in table 4 .



Figure 4. Plots of fluorescence lifetime of pyrene against concentration of PE4VP ${ }_{100}-\mathrm{PS}_{221}-\mathrm{PE} 4 \mathrm{VP}_{100}$, with different quaternization degrees. $\mathbf{\Delta} 30 \%$; - $75 \%$.

Table 4. Values of $\mathrm{cmc}$ obtained, by time-resolved fluorescence measurements, for copolymers forming crew-cut micelles.

\begin{tabular}{|c|c|c|}
\hline Copolymer & \% quaternization & $\mathrm{cmc}(\mu \mathrm{M})$ \\
\hline \multirow{2}{*}{ PE4VP-PS-PE4VP } & 30 & 0.45 \\
\cline { 2 - 3 } $67 / 106 / 67$ & 50 & 0.63 \\
\cline { 2 - 3 } & 65 & 0.78 \\
\hline \multirow{2}{*}{ PE4VP-PS-PE4VP } & 30 & 0.16 \\
\cline { 2 - 3 } $100 / 221 / 100$ & 50 & 0.34 \\
\cline { 2 - 3 } & 75 & 0.85 \\
\hline
\end{tabular}

Interestingly, the $\mathrm{cmc}$ are similar to those measured for star micelles but, in this case, the cmc increases with increasing charge density on the hydrophilic blocks. In other words, the cmc decreases in less hydrophilic copolymers. This behavior is similar to that reported for normal surfactant molecules.

Characterization of the aggregates. In spite of pyridinium ion is a very efficient quencher of pyrene fluorescence ${ }^{24}$, the lifetimes, measured at concentrations above the $\mathrm{cmc}$, are quite long even in copolymers with high degree of quaternization. This result suggests that pyrene must be located into the PS core, distant from the charged corona. For star micelles the micropolarity can be assessed by measuring the ratio $I_{1} / I_{3}$ in the fluorescence spectra of pyrene. In figure 5 are plotted the values of the ratio $\mathrm{I}_{1} / \mathrm{I}_{3}$ as a function of the concentration of PE4VP ${ }_{104}-\mathrm{PS}_{30}-\mathrm{PE} 4 \mathrm{VP}_{104}$ at different degrees of quaternization.



Figure 5. Plots of the ratio $\mathrm{I}_{1} / \mathrm{I}_{3}$ against concentration of $\mathrm{PE} 4 \mathrm{VP}_{104}-\mathrm{PS}_{30^{-}}$ PE4VP , with different degrees of quaternization: • $30 \%$; $\Delta 45 \%$; $70 \%$; $\boldsymbol{\nabla} 80 \%$. Dotted line is at concentration equal to $3.2 \mu \mathrm{M}$. 
At low copolymer concentration pyrene is in the aqueous phase and the values of the ratio $I_{1} / I_{3}$ are between 1.76 and 1.84. As the concentration increases the ratio decreases, indicating the transfer of pyrene from the aqueous phase to the hydrophobic environment provided by the copolymer. The final value of the ratio $I_{1} / I_{3}$ corresponds to the situation where all pyrene is located into the micelles. Therefore, a comparison of these values is a relative measure of the micelle micropolarity. In figure 5 the dotted line corresponds to $3.2 \mu \mathrm{M}$, which is higher than the $\mathrm{cmc}$ for all these systems. It is interesting to note that, at this concentration, the polarity sensed by pyrene decreases with increasing quaternization degree.

At the lowest percentage of quaternization only a slight decrease in the ratio $I_{1} / I_{3}$ is observed. This result indicates that for these micelles, pyrene is in a very polar environment, probably adsorbed to the surface of the PS core. In this location pyrene is in contact with water molecules but, at the same time protected from the pyridinium ions. For the copolymer with $80 \%$ of quaternization the ratio is as low as 1.15 , which indicates that pyrene has been incorporated into the hydrophobic PS core. This result could be attributed to the increased electrostatic repulsion in the micelle corona, due to the increase of the charge density in the PE4VP blocks. To diminish this repulsion the chains of the corona would extend themselves, allowing water molecules to enter into the corona. As a result pyrene is transferred into the more hydrophobic aggregate core. In other words, the increase of the electrostatic repulsion between the PE4VP blocks forming the micelle corona, enhance the inclusion of pyrene into the PS core. This effect is quite interesting and currently we are trying to demonstrate its existence in other copolymer systems.

Finally, the morphology and size of the aggregates were confirmed by transmission electronic microscopy (TEM). The images obtained for crewcut micelles formed by $\mathrm{PE}_{\mathrm{VVP}}-\mathrm{PS}_{106}-\mathrm{PE}_{6 \mathrm{VP}}$ at $65 \%$ of quaternization are shown in figure 6.



Figure 6. Images of crew-cut micelles, formed by PE4VP ${ }_{67}-\mathrm{PS}_{106}-\mathrm{PE} 4 \mathrm{VP}_{67}$ at $65 \%$ of quaternization, obtained by transmission electronic microscopy.

The pictures indicate that the aggregates are large spherical micelles with an average diameter of $413 \pm 72 \mathrm{~nm}$.

\section{CONCLUSIONS}

Cationic PE4VP-PS-PE4VP block copolymers were synthesized by sequential anionic polymerization, and reaction of the pyridine groups with ethyl bromide. In aqueous solution these copolymers aggregate to form star and crew-cut micelles. The cmc, determined by fluorescence probing methods, are in the order of $10^{-7} \mathrm{M}$. For crew-cut micelles the cmc values increases with increasing charge density of the PE4VP blocks. This behavior is similar to that observed in the formation of micelles by normal surfactant molecules. On the other hand, for copolymers forming star micelles there is not correlation between the quaternization degree and the $\mathrm{cmc}$.

The fluorescence lifetime of pyrene in aqueous solutions of these copolymers is rather long, despite the high local concentration in the corona of pyridinium ions, a very efficient quencher of pyrene emission. This result indicates that in all micelles pyrene is located in an environment where is protected from the pyridinium ions. The polarity of these micelles was evaluated by measurements of the ratio $\mathrm{I}_{1} / \mathrm{I}_{3}$. Interestingly, the polarity sensed by pyrene decreases with increasing charge density on the micelle corona. This effect is explained in terms of an enhanced transfer of pyrene from the aqueous phase into the PS core. The driving force for this transfer is the rearrangement of polymer chains in the micelle corona due to a largest electrostatic repulsion.

\section{ACKNOWLEDGMENTS}

This work was supported by FONDECYT 1070371, and Universidad Andrés Bello DI 53-06/R. I. Fuentes thanks Conicyt for a Doctoral Fellowship.

\section{REFERENCES}

1. M. J. Rosen, Surfactants and Interfacial Phenomena, John Wiley, New York, 1989.

2. C. Tanford, The Hydrophobic Effect, John Wiley, New York, 1988.

3. J. H. Fendler and E. J. Fendler, Catalysis in Micellar and Macromolecular Systems, Academic Press, New York, 1975.

4. J. H. Fendler, Membrane Mimetic Chemistry, John Wiley, New York, 1982.

5. Alexandridis P. and Lindman B., Amphiphilic Block Copolymers, SelfAssembly and Applications, Elsevier Science, Amsterdam, 2000.

6. L. F. Zhang and A. Eisenberg Journal of the American Chemical Society, 118, 3168 (1996).

7. L. F. Zhang and A. Eisenberg Macromolecules, 32, 2239 (1999).

8. I. Astafieva, K. Khougaz, and A. Eisenberg Macromolecules, 28, 7127 (1995).

9. H. W. Shen and A. Eisenberg J.Phys.Chem.B, 103, 9473 (1999).

10. L. F. Zhang and A. Eisenberg Macromolecules, 29, 8805 (1996).

11. Y. S. Yu, L. F. Zhang, and A. Eisenberg Macromolecules, 31, 1144 (1998).

12. A. V. Kabanov and V. Y. Alakhov, in Amphiphilic Block Copolymers. Self-assembly and applications, P. Alexandridis and B. Lindman, Ed., Elsevier. Amsterdam 2000.

13. M. Malstem, in Amphiphilic Block Copolymers. Self-assembly and applications, P. Alexandridis and B. Lindman, Ed., Elsevier. Amsterdam 2000.

14. Hadjichristidis N., Pispas S., and Floudas G., Block Copolymers, Synthetic Strategies, Physical Properties, and Applications, John Wiley \& Sons, Inc., Hoboken, New Jersey, 2003.

15. Z. S. Gao, S. K. Varshney, S. Wong, and A. Eisenberg Macromolecules, 27, 7923 (1994)

16. C. L. Zhao, M. A. Winnik, G. Riess, and M. D. Croucher Langmuir, 6, 514 (1990).

17. A. F. Olea, R. G. Barraza, I. Fuentes, B. Acevedo, and F. Martinez Macromolecules, 35, 1049 (2002).

18. C. H. Salamanca, R. G. Barraza, B. Acevedo, and A. F. Olea J.Chil.Chem. Soc, 52, 1025 (2007).

19. K. Kalyanasundaram and J. K. Thomas J.Am.Chem.Soc., 99, 2039 (1977).

20. A. F. Olea, B. Acevedo, and F. Martinez J.Phys.Chem.B, 103, 9306 (1999).

21. K. Kalyanasundaram, Photochemistry in Microheterogeneous Systems, Academic Press, Orlando, 1987.

22. M. Wilhelm, C. L. Zhao, Y. C. Wang, R. L. Xu, M. A. Winnik, J. L. Mura, G. Riess, and M. D. Croucher Macromolecules, 24, 1033 (1991).

23. E. A. Lysenko, T. K. Bronich, E. V. Slonkina, A. Eisenberg, V. A. Kabanov, and A. V. Kabanov Macromolecules, 35, 6351 (2002).

24. H. Rosenbluth, B. Weiss-Lopez, and A. F. Olea Photochem.Photobiol., 66, 802 (1997). 